\title{
Collateral benefit in pulmonary atresia with ventricular septal defect?
}

\author{
S. Ram Kumar, MD, PhD, FACS
}

\footnotetext{
From the Division of Cardiac Surgery, Department of Surgery, University of Southern California and Heart Institute, Children's Hospital of Los Angeles, Los Angeles, Calif.

Disclosures: Author has nothing to disclose with regard to commercial support.

Received for publication April 8, 2018; accepted for publication April 9, 2018; available ahead of print May 9 , 2018.

Address for reprints: S. Ram Kumar, MD, PhD, FACS, Children's Hospital, Los Angeles, 4650 Sunset Blvd, Mailstop \#66, Los Angeles, CA 90027 (E-mail: rsubramanyan@ @hla.usc.edu).

J Thorac Cardiovasc Surg 2018;156:1205-6

$0022-5223 / \$ 36.00$

Copyright (c) 2018 by The American Association for Thoracic Surgery

https://doi.org/10.1016/j.jtcvs.2018.04.030
}

Pulmonary atresia with ventricular septal defect (VSD) is a complex congenital heart defect that can manifest with a variable clinical picture driven largely by the heterogeneity in the anatomy of the central pulmonary arteries (PAs). During early development, a single outflow tract exits from the developing right ventricle (RV). This outflow tract is then septated and aligned such that each ventricle is connected via a semilunar valve to the aorta or PA. An aberrant septation process leading to extreme anterior deviation of the conotruncal septum results in a malalignment VSD with an atretic RV outflow. Developmental derangements can also extend into the pharyngeal arches. Frequently, the left fourth pharyngeal arch artery inappropriately regresses, resulting in a right-sided aortic arch that can be associated with a right aortic descent (Figure 1). Involvement of the sixth arch leads to anomalies in the PA and ductus arteriosus.

In its most simplistic form, VSD/pulmonary atresia is accompanied by confluent branch PA fed by a good-sized ductus. Management of this condition is relatively straightforward and involves closing the VSD and establishing continuity between the RV and branch PA. When ductal flow is interrupted during development, it has been postulated that decreased flow through the central PA impairs appropriate branching and maturation, resulting in inadequate perfusion to segments of the lung. The resultant hypoxia serves as a stimulus for enhanced angiogenesis from neighboring systemic blood vessels, leading to the formation of major aortopulmonary collateral arteries (MAPCAs, Figure 1). Areas of the lung may receive additional blood supply from MAPCA (dual supply) or rely exclusively on MAPCA for perfusion. In the most extreme form, the central PAs essentially do not form and pulmonary perfusion is exclusively from MAPCA.

Early reports indicated that long-term survival is uniformly poor when pulmonary blood flow is exclusively from MAPCAs. ${ }^{1}$ This spurred an interest in surgical

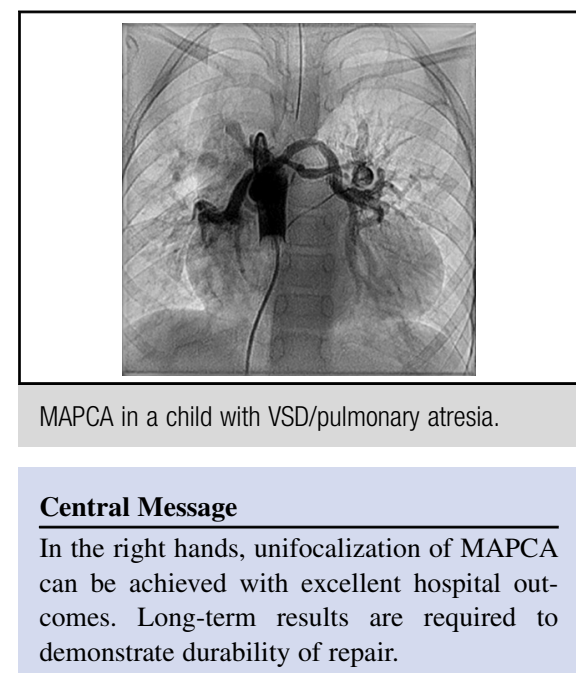

See Article page 1194.

palliation. Despite several optimistic reports, the appropriate management of the more complex form of VSD/pulmonary atresia with MAPCA continues to be controversial, at least in part due to philosophical differences related to its pathophysiology. It has been proposed that MAPCAs represent dilated bronchial vessels, ${ }^{2}$ which are fundamentally different from PAs and are incapable of growth required to perfuse the developing lung.

Therefore, some surgeons favor rehabilitating the native central PA. ${ }^{4}$ Such a strategy is contingent on the expectation that the native PA, albeit diminutive, can be consistently identified in all patients with VSD/pulmonary atresia. It also relies on effective collateralization of the native PA to provide flow to an adequate number of pulmonary segments. In contrast, other groups have favored the midline "unifocalization" approach, translocating important MAPCAs from their systemic origin to RV outflow. ${ }^{5}$ It has been suggested that such an approach can be more universally applied even when the central PA cannot be identified. ${ }^{6}$

In this issue of the Journal, Mainwaring and colleagues, ${ }^{7}$ who have championed the cause of primary unifocalization, present their surgical algorithm applied to 307 patients who underwent primary treatment at Stanford. The results are impressive. More than $90 \%$ of the patients have reached biventricular circulation, with an overall mortality of about $9 \%$ and an on-table RV pressure that was less than $40 \%$ systemic. Two-thirds of patients were repaired in a single- 


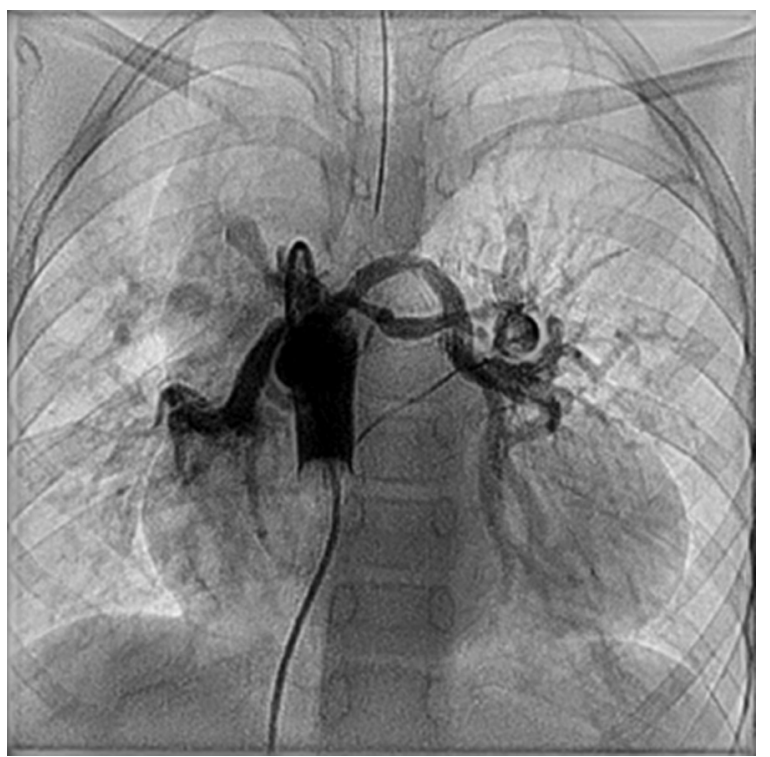

FIGURE 1. Angiogram showing multiple aortopulmonary collaterals arising from the descending thoracic aorta in a child with pulmonary atresia and ventricular septal defect.

stage procedure. These results bear testimony to what can be achieved by a team of talented physicians who focus their efforts on a challenging clinical problem. Such outcomes create hope for families and physicians who deal with the difficult consequences of these complex defects.

That said, how generalizable are these results? This study does not provide us data on the hospital course following these technically demanding procedures. It is conceivable that there is a significant intellectual and resource burden associated with the care of these patients, which may not be reproducible at all centers. In addition, no data are provided on the fate of the reconstructed MAPCAs over time. The short follow-up in the entire cohort precludes us from discounting the argument that the eventual contribution of translocated MAPCAs to pulmonary circulation is negligible. ${ }^{3}$ Other questions also remain -is there a minimum number of lung segments with PA flow for adequate repair? What is the best approach to rehabilitate diminutive native PA — central shunt, direct implantation into aorta, or percutaneous dilation? It has been suggested that these vessels may have a greater growth potential early in life-so is there an ideal time when repair should be offered?

Despite the heterogenous nature of the disease process and seemingly divergent treatment paradigms, several common themes emerge from these outstanding reports. ${ }^{7}$ The ultimate goal in VSD/pulmonary atresia with MAPCAs is to achieve biventricular circulation with low RV and PA pressures, with the PA supplying as many segments of the lung as feasible. When there is dual supply, rehabilitating the native PA leads to reliable growth and a favorable outcome. Patients with MAPCAs who present with high oxygen saturation indicating good pulmonary circulation represent a favorable subgroup that can usually undergo a single-stage unifocalization and complete repair. For the subset of patients who lack adequate blood supply from the native PA, we await longer-term results from centers of excellence to better understand the "unnatural" history of the unifocalized MAPCAs.

\section{References}

1. Bull K, Somerville J, Ty E, Spiegelhalter D. Presentation and attrition in complex pulmonary atresia. J Am Coll Cardiol. 1995;25:491-9.

2. Nørgaard MA, Alphonso N, Cochrane AD, Menahem S, Brizard CP, d'Udekem Y. Major aorto-pulmonary collateral arteries of patients with pulmonary atresia and ventricular septal defect are dilated bronchial arteries. Eur J Cardiothorac Surg. 2006;29:653-8.

3. d'Udekem Y, Alphonso N, Nørgaard MA, Cochrane AD, Grigg LE, Wilkinson JL, et al. Pulmonary atresia with ventricular septal defects and major aortopulmonary collateral arteries: unifocalization brings no long-term benefits. J Thorac Cardiovasc Surg. 2005;130:1496-502.

4. Liava'a M, Brizard CP, Konstantinov IE, Robertson T, Cheung MM, Weintraub R, et al. Pulmonary atresia, ventricular septal defect, and major aortopulmonary collaterals: neonatal pulmonary artery rehabilitation without unifocalization. Ann Thorac Surg. 2012;93:185-91.

5. Malhotra SP, Hanley FL. Surgical management of pulmonary atresia with ventricular septal defect and major aortopulmonary collaterals: a protocol-based approach. Semin Thorac Cardiovasc Surg Pediatr Card Surg Annu. 2009;145-51.

6. Carrillo SA, Mainwaring RD, Patrick WL, Bauser-Heaton HD, Peng L, Reddy VM, et al. Surgical repair of pulmonary atresia with ventricular septal defect and major aortopulmonary collaterals with absent intrapericardial pulmonary arteries. Ann Thorac Surg. 2015;100:606-14.

7. Mainwaring RD, Patrick WL, Roth SJ, Kamra K, Wise-Faberowski L, Palmon M, et al. Surgical algorithm and results for repair of pulmonary atresia with ventricular septal defect and major aortopulmonary collaterals. J Thorac Cardiovasc Surg. 2018;156:1194-204. 